\section{Arzneiausgaben 2012 fast stabil}

Keine außergewöhnlichen Schwankungen bei den Azneimittelausgaben, so das IMS Health. Dennoch ist ein Zahlenvergleich von Interesse.

D ie Arzneimittelausgaben sind 2012 Millionen $€$ (plus 1,5\%) gestiegen, berichtet das Marktforschungsunternehmen IMS Health. Insgesamt haben Vertragsärzte damit Arzneimittel und TestDiagnostika im Wert von 29,4 Milliarden $€$ zulasten der Krankenkassen verordnet. Der Ausgabenanstieg liegt signifikant unter der Rahmenvereinbarung von Krankenkassen und KBV für 2012, die eine Quote von 2,7\% vorgesehen hatte. Zwangsrabatte von Herstellern und Apotheken sind dabei berücksichtigt, nicht aber Einsparungen aus Rabattverträgen und Patientenzuzahlungen. Die Zahl abgegebener Packungen hat sich geringfügig um 0,1\% auf 677 Millionen Packungen verringert. Zugenommen hat dabei allerdings der Anteil größerer Packungen, und zwar um 3,7\% (N3). Kleinere Packungen dagegen wurden entsprechend seltener verordnet. den $€$. gehen. im Vergleich zum Vorjahr um 443

Die Höhe der Zwangsrabatte zugunsten von GKV und PKV hat sich 2012 nur wenig im Vergleich zum Vorjahr verändert. Hersteller mussten den gesetzlichen Krankenassen Nachlässe von 2,503 (2011: 2,526) Milliarden $€$ gewähren. PKV-Unternehmen profitierten von Rabatten im Umfang von 440 (2011: 446) Millionen $€$. Die Abschläge der Apotheker beliefen sich auf 1,252 (2011: 1,249) Milliarden $€$. Insgesamt addierten sich die Zwangsrabatte somit auf 4,2 Milliar-

Im Dezember 2012 ergibt sich ein ungewöhnlicher Ausgabenrückgang um 6,5\%. Die Menge verordneter Packungen sank sogar um $7,7 \%$. Vermutlich haben Patienten mit Blick auf das Auslaufen der Praxisgebühr den Arztbesuch aufgeschoben. Denn die Rückgänge betreffen auch Indikationen, die gewöhnlich mit einer Dauermedikation einher-

Florian Steack

\section{Rekordwerte für das Vertrauen in das Gesundheitssystem}

\section{Vertrauen Ärzte und Gesamtbevölkerung weiterhin auf die Leistungsfähigkeit des deutschen Gesundheitssystems?}

$\mathrm{N}$ ach einer Repräsentativumfrage des Instituts für Demoskopie Allensbach im Auftrag des MLP-Gesundheitsreports beurteilen $93 \%$ der Ärzte und $82 \%$ der Gesamtbevölkerung die Gesundheitsversorgung 2012 als gut oder sogar sehr gut. Die Werte sind seit 2008 kontinuierlich gestiegen. Renate Köcher vom Allensbach-Institut nennt als Ursache, dass die Bürger derzeit nicht mit Reformdiskussionen verunsichert werden.

Nach wie vor ist Gesundheitspolitik trotz etwas besserer Werte als 2009 kein Feld, mit dem die Bundesregierung trumpfen könnte: lediglich $18 \%$ (2009: $4 \%$ ) der Bürger haben hier einen guten Eindruck. Ähnliches spiegelt sich in der den Parteien zugebilligten gesundheitspolitischen Kompetenz wider: Im Urteil der Bürger kommen Union und SPD mit jeweils 15\% (Anteil derer, die den Parteien Ideen für ein vernünftiges gesundheitspolitisches Konzept zubilligen) auf die besten Werte. Die Grünen liegen bei $6 \%$, die Linke bei $5 \%$, die FDP bei $3 \%$. Lediglich bei Ärzten schneidet die FDP mit $12 \%$ besser ab.

Insgesamt sagen inzwischen $69 \%$ der Ärzte, dass die Attraktivität des Arztberufs wächst (2010: 57\%). Als gut oder sehr gut beurteilen $82 \%$ der Hausärzte ihre Situation (2011: 76\%). Bei den Fachärzten sinkt der Anteil von $81 \%$ auf $72 \%$. $62 \%$ der Ärzte sind mit der letzten Honorarrunde unzufrieden; die Honorarverhandlungen in den meisten KVen sind allerdings noch nicht abgeschlossen.

Helmut Laschet
BMG wehrt sich gegen weitere Kürzung der Gesundheitsfonds

Das BMG wehrt sich gegen Pläne des Finanzministeriums, die Zuschüsse für den Gesundheitsfonds weiter zu senken. „Wir haben unseren Sparbeitrag mit 4,5 Milliarden $€$ bereits geleistet. Jetzt sind andere Ressorts gefragt ", so das BMG. 2013 soll der Zuschuss von 14 Milliarden $€$ um 2,5 Milliarden $€$ gekürzt werden, 2014 um 2 Milliarden $€$. Medienberichten zufolge will die Bundesregierung den Zuschuss 2014 sogar um 4 Milliarden kürzen. Dies hat eine Sprecherin des Finanzministeriums als "so inhaltlich nicht zutreffend" zurückgewiesen. Begehrlichkeiten wecken die vollen Sozialkassen gleichwohl: Die Überschüsse seien ein Spiegelbild der guten Konjunktur. „Es macht wenig Sinn, ein Defizit im Haushalt und gleichzeitig hohe Überschüsse in der Gesundheitskasse zu haben", sagte der Staatssekretär im Bundesfinanzministerium, Steffen Kampeter (CDU). sun/af

\section{Bedingtes Ja zum Sicherstellungsauftrag}

Rund drei Viertel der Vertragsärzte und Vertrags-Psychotherapeuten bejahen den Sicherstellungsauftrag, $66 \%$ verknüpfen dies aber mit der Forderung nach weitreichenden Reformen. Lediglich 6\% wollen einen Systemwechsel. Dies ist das Ergebnis eines Referendums unter den rund 149.000 niedergelassenen Ärzten und Psychotherapeuten, das von der KBV-VV im September 2012 beschlossen worden war. $53 \%$ hatten sich daran beteiligt.

Feste und kostendeckende Preise für ärztliche Leistungen fordern $93 \%$. Gleich stark ist das Votum, Form und Inhalte ausschließlich von der ärztlichen Selbstverwaltung und nicht von den Krankenkassen bestimmen zu lassen. $91 \%$ sprechen sich gegen eine Honorarabstaffelung als Methode der Mengensteuerung aus. $85 \%$ plädieren dafür, dass die Verantwortung für diagnostische und therapeutische Freiheit allein bei der ärztlichen Selbstverwaltung liegen soll. Ähnlich stark ist das Votum für den Vorrang der ambulanten Medizin. Etwas schwächer ausgeprägt (71\%) ist die Forderung nach Abschaffung von Regressen wegen Arznei- und Heilmittelverordnungen.

Das Referendum belegt eine hohe intrinsische Motivation der niedergelassenen Ärzte. $92 \%$ bezeichnen ihre Arbeit als „sinnvoll“. $72 \%$ wünschen sich mehr Zeit für die direkte Zuwendung zu Patienten.

un/af 